\title{
Vaspin (but not neuropeptide B or neuropeptide W) as a possible predictor of body weight normalization in anorexia nervosa
}

\author{
Teresa Grzelak ${ }^{1}$, Marta Tyszkiewicz-Nwafor ${ }^{2}$, Agata Dutkiewicz' ${ }^{2}$ Aniceta Ada Mikulska ${ }^{3}$, \\ Monika Dmitrzak-Weglarz ${ }^{4}$, Agnieszka Slopien², Krystyna Czyzewska ${ }^{3}$, Elzbieta Paszynska ${ }^{5}$
}

\begin{abstract}
'Department of Physiology, Poznan University of Medical Sciences, Poznan, Poland 2Department of Child and Adolescent Psychiatry, Poznan University of Medical Sciences, Poznan, Poland

${ }^{3}$ Division of Biology of Civilization-Linked Diseases, Department of Chemistry and Clinical Biochemistry, Poznan University of Medical Sciences, Poznan, Poland

${ }^{4}$ Psychiatric Genetics Unit, Department of Psychiatry, Poznan University of Medical Sciences, Poznan, Poland

${ }^{5}$ Department of Integrated Dentistry, Community Dentistry Section, Poznan University of Medical Sciences, Poznan, Poland
\end{abstract}

Submitted: 12 September 2017; Accepted: 4 December 2017;

Online publication: 9 April 2018

Arch Med Sci 2021; 17 (2): 376-381

DOI: https://doi.org/10.5114/aoms.2018.74969

Copyright (c) 2018 Termedia \& Banach

\begin{abstract}
Introduction: The aim of the study was to evaluate the correlation between the nutritional status of patients with anorexia nervosa (AN) and levels of vaspin (VASP), neuropeptide B (NPB), neuropeptide $W(N P W)$ and total antioxidant status (TAS).

Material and methods: Ninety serum samples collected from 30 teenage female patients during the acute stage of AN and 30 healthy persons (CONTR) were subjected to biochemical analysis; patients with AN were examined at the beginning of the study (AN-I) and after hospitalization (AN-II), as a result of which partial stabilization of anthropometric measurements was achieved (an increase of body mass index (BMI) by $3.5 \mathrm{~kg} / \mathrm{m}^{2}$ ).

Results: Vaspin levels dropped at the end of the hospitalization (compared to AN-I, $p<0.05$ ), achieving values comparable to the CONTR; moreover there was a positive correlation between VASP level and the achieved body weight in AN-II $(p<0.05)$. Positive correlations were also noted with regard to VASP vS. NPB in AN-I ( $p<0.02)$ (and AN-II, $p<0.013)$, as well as in the case of VASP vs. NPW in the same groups ( $p<0.02$ and $p<0.015$, respectively). NPB concentration was higher in AN-I $(p<0.05)$ and AN-II $(p<0.018)$ than in CONTR, whereas there were no differences $(p>0.05)$ with regard to levels of VASP, NPW, or TAS. Conclusions: The high level of NPB despite treatment and normalization of VASP level may suggest that there are chronic neuroendocrine disorders at play in anorexia nervosa.
\end{abstract}

Key words: anorexia nervosa, vaspin, neuropeptide B, neuropeptide W, antioxidant status.

\section{Introduction}

Anorexia nervosa (AN) is a disease entity the etiology of which is not fully understood and its progression is difficult to foresee [1-5]. Aside from mental and social conditions, neuroendocrine factors become more and more emphasized in the development of AN. These factors signifi-

\author{
Corresponding author: \\ Teresa Grzelak PhD \\ Department of Physiology \\ Poznan University \\ of Medical Sciences \\ 6 Swiecickiego St \\ 60-871 Poznan, Poland \\ Phone/Fax: +48 618546540 \\ E-mail: tgrzelak@ump.edu.pl
}


cantly influence the course of the disease and may be important from the point of view of therapy and mortality [6-8]. Vaspin (VASP) is a serine protease inhibitor. There are suggestions that this molecule stabilizes insulin as it is also a kallikrein 7 antagonist that inhibits the activity of insulin [9]. Vaspin is produced both in the visceral adipose tissue and in the subcutaneous adipose tissue, as well as in the cells of the hypothalamus, pancreas, stomach, skin and cerebrospinal fluid [10]. There are few studies on the concentration of this adipocytokine in malnourished patients. von Loeffelholz et al. noted higher VASP levels in groups of people with normal body weight compared to an underweight population and obese population [11].

Neuropeptide B (NPB) and neuropeptide W (NPW) are endogenous ligands of GPR7 and GPR8 receptors that belong to the family of GPCR receptors (G-protein-coupled receptors) responsible for the intake of food (they take part in energy homeostasis) and regulation of the hypothalamic-pituitary-adrenal axis [12]. The highest mRNA expression for NPB was noted in substantia nigra, spinal cord, hypothalamus, hippocampus, stomach, spleen, placenta, testicles, and ovaries [13]. Relatively high amounts of mRNA for NPW were noted in human brain, hippocampus, substantia nigra, stomach, and rectum [14], whereas the expression of neuropeptide $W$ in the stomach depended on the level of nutrition and the type of consumed foods [15]. However, there are no data from research on neuropeptides $B$ and $W$ in persons with anorexia nervosa.

The aim of the present study was to evaluate the correlation between the nutritional status of female patients with anorexia nervosa (AN) and the concentrations of VASP, neuropeptide B (NPB), and neuropeptide W (NPW), and total antioxidant status (TAS) in serum.

The null hypothesis stated that there are no differences in serum levels of VASP, NPB, NPW, and TAS between the analyzed groups (AN-I, AN-II, CONTR). The alternative hypothesis was that the serum levels of VASP, NPB, NPW, and TAS in groups with anorexia nervosa (AN-I and $A N-I I)$ and the control group (CONTR) are different.

\section{Material and methods}

\section{Characteristics of the studied population}

The Local Bioethics Committee has given its permission to conduct the research project (No. $329 / 15)$. Participation in the analysis was voluntary, and each participant and their legal guardian signed an informed consent form regarding participation in the project after having been informed about the project's purpose and course. The study was carried out in accordance with the Declaration of Helsinki.
Biochemical analyses were performed on 90 serum samples collected from 30 teenage female patients with severe AN (AN, showing all of the symptoms of restrictive or bingeing-purging subtype of AN, including amenorrhea, body mass index (BMI; < $15 \mathrm{~kg} / \mathrm{m}^{2}$ ) and 30 healthy peers (CONTR) aged 12-18; patients with AN were examined at the beginning of the study $(A N-I)$ and at the end of the hospital treatment (AN-II) when they gained the desired body weight (taking into account age, height, and the observed BMI increase of $3.5 \pm 0.5$ $\mathrm{kg} / \mathrm{m}^{2}$ ). Men were excluded from the study due to the small number of adolescent males hospitalized in the Department of Child and Adolescent Psychiatry because of anorexia nervosa. The analysis involved checking levels of VASP, NPB, NPW, and TAS in samples. Two independent psychiatrists from the Department of Child and Adolescent Psychiatry confirmed the diagnosis of severe anorexia nervosa on the basis of WHO guidelines (ICD 10 (code F 50.1)) [16]. Along with psychological therapy, and sometimes also treatment with standard pharmaceuticals, the patients with anorexia nervosa were provided treatment (including nutrition) during their hospital stay to reduce the risk of fatal somatic complications. At the same time, some of the patients received pharmacological treatment, including antidepressants for patients who exhibited depressed mood and/or anxiolytic drugs for patients who exhibited some symptoms of anxiety disorders, especially obsessions and/or compulsions. Average hospitalization time for the patients was $76 \pm 10$ days.

In the AN and CONTR groups anthropometric measurements were taken after overnight fasting (body weight and height analyses with accuracy to $0.1 \mathrm{~kg}$ (certified weight) and to $0.1 \mathrm{~cm}$ (stadiometer) in light underwear, with no shoes). On the basis of these analyses the following calculations were made: body mass index, body mass index z-score and body mass index-for-age percentile taking into account norms for females and the height of Polish teenagers [17]. The control group consisted of healthy females of a similar age, height and education level as members of the AN group. Exclusion criteria in the control group included: abnormal body weight, poor health (mental and somatic), including the presence of eating disorders (also in the past and concerning first-level relatives), and addiction to psychoactive substances, according to physical examination and laboratory analyses, following a detailed interview.

\section{Biochemical analyses}

Samples of venous blood (about $5 \mathrm{ml}$ ) were collected from each study participant after overnight fasting and an all-night rest between 7:00 
and 8:00 in the morning. Part of the blood samples was subjected to biochemical analyses immediately after collection, whereas another part of the samples, required to assay proteins and other molecules, was appropriately secured and frozen at $-80^{\circ} \mathrm{C}$. The analysis of VASP (Mediagnost, Reutlingen, Germany) and neuropeptides $B$ and W (Sunred Biological Technology, Shanghai, China) concentration in serum was carried out closely in accordance with the guidelines of the manufacturer, using commercial immunoenzymatic tests. Microtiter plates with the fixed primary antibody (specific for the determined molecules) were incubated with serum (containing the antigen, namely the measured protein) and later with a secondary antibody marked with peroxidase.

Subsequently, a reaction was provoked with a substrate for chromogen and the color was monitored on an ELISA MR-96 microplate reader manufactured by CLINDIAG SYSTEMS B.V.B.A. (Pollare, Belgium). Protein levels were calculated on the basis of calibration curves which were determined using a 4-parameter-algorithm (SigmaPlot 11.00 software) prepared each time for a specific set of assays. Intra-assay and inter-assay coefficients of variation (CVs) were respectively for VASP $2.4 \%$ and $3.0 \%$, for NPB $3.4 \%$ and $6.0 \%$, and for NPW $4.2 \%$ and $6.2 \%$.

Serum TAS was assayed using a test by Randox Laboratories Ltd (Crumlin, UK). TAS analysis involved incubation of the patented molecule 2,2-azino-bi-[3-ethylbenzthiazoline sulfonate] (ABTS) with peroxidase (metmyoglobin) and hydrogen peroxide, which produced an ABTS+ radical cation with characteristic color detected at wavelength 600 nm (using an ELISA MR-96 microplate reader by CLINDIAG SYSTEMS B.V.B.A. (Pollare, Belgium)). Intra-assay CV for TAS was $4.0 \%$ and inter-assay CV was $5.9 \%$. TAS results were presented as the Trolox equivalent [mmol/l] [18].

\section{Statistical analysis}

The results were then subjected to statistical analysis using Statistica 12.5 software with Medical Set (StatSoft Inc., USA)., including, among other things, analyses for comparing medians for related (AN-I vs. AN-II; using the Wilcoxon test) and unrelated date (AN-I vs. CONTR, AN-II vs. CONTR; Mann-Whitney $U$ test and Student's $t$-test or Welch's ANOVA test for unequal variances, and correlation between the studied variables (Spearman's test for nonparametric data and Pearson's test for parametric data). Normality of data distribution was checked using the Shapiro-Wilk test. Homogeneity of variance was confirmed using Levene's test. The level of statistical significance was set at $p<0.05$.

\section{Results}

Patients with severe anorexia nervosa (AN-I) before the treatment and after 76 days of hospitalization (AN-II) in the Department of Child and Adolescent Psychiatry were found to have significantly lower levels of anthropometric measurements and neuropeptide B compared to the CONTR group (Table I). In patients with anorexia nervosa median levels of VASP were lower at the end of the hospitalization (by 33\%, $p<0.05$, AN-II) compared to the levels before treatment (AN-I), achieving values similar to those in the CONTR group, whereas as regards the level of this adipocytokine in the $\mathrm{AN}$-II group there was a positive correlation between the obtained body weight $(r=0.36, p<0.05)$ and the change in TAS in this cohort of patients (comparing the status before and after hospital treatment); a correlation was observed between the initial body weight ( $r=$ $0.48, p<0.008)$ and the change in TAS as well as a correlation between body weight after the treatment and the change in TAS $(r=0.39, p<0.04$, Table II).

Positive correlations were noted between values of VASP and NPB in the AN-I $(n=30 ; r=$ $0.46 ; p<0.02)$ and AN-II $(n=30 ; r=0.45 ; p<$ $0.013)$ groups, as well as between concentrations of VASP and NPW in the same groups, respectively $(n=30 ; r=0.54 ; p<0.02 ; n=30 ; r=0.44$; $p<0.015)$. Also, positive correlations existed between NPB and NPW in the above groups: AN-I $(n=30 ; r=0.95 ; p<0.000001), \mathrm{AN}-\mathrm{II}(n=30$; $r=0.97 ; p<0.000001$, Table II) and CONTR $(n=30$; $r=0.90 ; p<0.0005)$.

\section{Discussion}

As expected, the nutritional status of patients with anorexia nervosa has improved as a result of the hospital treatment, but the patients' body weight did not achieve normal levels, as shown in cohort studies and meta-analysis [2, 19]. High concentrations of VASP in the group of studied patients with severe anorexia nervosa and the decrease of this parameter after hospitalization (to levels similar to those in the control group) when BMI increased by $3.5 \mathrm{~kg} / \mathrm{m}^{2}$ on average suggests that this adipocytokine influences the process of body weight normalization in the treatment of anorexia nervosa (therefore the null hypothesis was rejected). This correlation is also supported by the observed link between levels of VASP after hospitalization and the weight gained. However, test results concerning the level of this adipocytokine in underweight persons are inconclusive. Research by Oswiecimska et al. in 2016 indicated that there is a connection between VASP levels in serum and anorexia nervosa. In that case median 
Table I. Anthropometric and biochemical characteristics of persons, divided into groups with anorexia nervosa (AN-I and AN-II) and a control group (CONTR)

\begin{tabular}{|c|c|c|c|c|}
\hline Parameter [unit] & AN-I $(n=30)$ & AN-II $(n=30)$ & CONTR $(n=30)$ & $P$-value \\
\hline Age [years] & $16.0\{14.0 ; 17.0\}$ & $16.0\{14.0 ; 17.0\}$ & $15.0\{14.0 ; 17.0\}$ & $\begin{array}{l}N S^{*} \\
N S^{* *} \\
N S^{* * *}\end{array}$ \\
\hline Body mass [kg] & $36.0\{33.0 ; 40.0\}$ & $45.6\{39.5 ; 49.1\}$ & $54.0\{49.6 ; 58.0\}$ & $\begin{array}{l}<0.000003^{*} \\
<0.00007^{* *} \\
<0.0001^{* * *}\end{array}$ \\
\hline High [m] & $1.61\{1.55 ; 1.66\}$ & $1.61\{1.55 ; 1.66\}$ & $1.64\{1.62 ; 1.68\}$ & $\begin{array}{c}N^{*} \\
<0.031^{* *} \\
<0.031^{* * *}\end{array}$ \\
\hline BMI $\left[\mathrm{kg} / \mathrm{m}^{2}\right]$ & $14.20\{13.67 ; 14.65\}$ & $17.50\{17.01 ; 18.11\}$ & $20.58\{17.91 ; 21.86\}$ & $\begin{array}{l}<0.000003^{*} \\
<0.0012^{* *} \\
<0.002^{* * *}\end{array}$ \\
\hline BMI z-score & $\begin{array}{c}-3.980\{-4.550 \\
-3.370\}\end{array}$ & $\begin{array}{c}-1.295\{-1.440 \\
-1.010\}\end{array}$ & $\begin{array}{c}-0.240 \\
\{-0.790 ; 0.450\}\end{array}$ & $\begin{array}{l}<0.000003^{*} \\
<0.0006^{\star *} \\
<0.000001\end{array}$ \\
\hline $\begin{array}{l}\text { Body mass index - } \\
\text { for - age percentile }\end{array}$ & $0.10\{0.10 ; 0.10\}$ & $11.0\{7.00 ; 18.00\}$ & $41.00\{21.00 ; 67.00\}$ & $\begin{array}{l}<0.000004^{*} \\
<0.0007^{\star *} \\
<0.000001\end{array}$ \\
\hline Vaspin [ng/ml] & $0.766\{0.424 ; 1.386\}$ & $0.578\{0.345 ; 0.935\}$ & $0.536\{0.294 ; 1.255\}$ & $\begin{array}{c}<0.05^{\star} \\
N S^{\star *} \\
N S^{\star * \star}\end{array}$ \\
\hline Neuropeptide B [ng/ml] & $0.295\{0.147 ; 0.888\}$ & $0.335\{0.172 ; 0.882\}$ & $0.159\{0.110 ; 0.295\}$ & $\begin{aligned} & N S^{*} \\
< & 0.05^{* \star} \\
< & 0.018^{* * *}\end{aligned}$ \\
\hline Neuropeptide W [ng/ml] & $0.205\{0.147 ; 0.359\}$ & $0.219\{0.143 ; 0.548\}$ & $0.161\{0.147 ; 0.221\}$ & $\begin{array}{l}N S^{*} \\
N S^{\star \star} \\
N S^{\star \star \star}\end{array}$ \\
\hline TAS $[\mathrm{mmol} / \mathrm{l}]$ & $1.037\{0.746 ; 1.156\}$ & $0.996\{0.816 ; 1.165\}$ & $1.092\{0.898 ; 1.266\}$ & $\begin{array}{l}N S^{*} \\
N S^{* *} \\
N S^{* * *}\end{array}$ \\
\hline
\end{tabular}

Parameters are shown as medians $\{25 \%$ and $75 \%$ quartiles\}, $n$ - number of persons, $A N-I-$ patients with anorexia nervosa in case of acute stage, AN-II - patients with anorexia nervosa after hospitalization, $p$ - level of statistical significance in Mann-Whitney test for nonparametric data or Student's t-test for parametric data (Welch's ANOVA test for variance date) in the case of unpaired data (AN-I vs. CONTR; AN-II vs. CONTR) or Wilcoxon test in the case of paired date, NS - difference not statistically significant, TAS - total antioxidant status, BMI-body mass index; BMI z-score-body mass index z-score, ${ }^{*} p$-value in case of AN-I vs. AN-Il analysis; ${ }^{* *} p$-value in case of $A N$-I vs. CONTR analysis, ${ }^{* * *} p$-value in case of $A N-I /$ vs. CONTR analysis.

VASP concentrations were higher in emaciated teenagers (BMI ranging between 11.4 and 17.3 $\mathrm{kg} / \mathrm{m}^{2}$ ) compared to their peers with BMI between 16.7 and $24.6 \mathrm{~kg} / \mathrm{m}^{2}$ [20], similarly as in our study. Additionally, patients with anorexia nervosa had high serum levels of this adipocytokine in a study by Ostrowska et al. [21]. Such results are also similar to the results obtained in the analyses of saliva (the research showed higher VASP levels in the saliva of teenagers with severe anorexia nervosa compared to the control group) [22]. Contrary to the above trends, the analyses concerning young children (average age 2 years) with level 2 and 3 malnourishment (caused by appetite disturbances) revealed lowered VASP levels in comparison to the group of peers with normal body weight [23].

In the group of patients with anorexia nervosa before and after several weeks of hospitalization, NPB levels in serum were found to be high com- pared to their healthy peers (therefore, the null hypothesis was again rejected). The maintenance of a pathological attitude towards food among anorexia nervosa patients depends on multiple neurological, endocrine and psychological factors [4]. Perhaps the clinical condition of anorexia nervosa also depends, among other things, on the level of NPB in the serum, but there are not enough direct data concerning its influence on the human body. In the case of animals (rodents) administration of this molecule in concentrations of $3 \mathrm{nmol} / \mathrm{l}$ and $10 \mathrm{nmol} / \mathrm{l}$ to cerebral ventricles in the daylight phase did not change the amount of consumed food, but in the night-time phase and in large doses it reduced food intake, whereas in a low dose it increased food intake for the first $2 \mathrm{~h}$ and then had a pro-anorexic effect [24]. Male NPB knockout animals were characterized by higher body weight (despite consumption of similar amounts of food and similar activity) compared to wild animals of 
Table II. Indices of correlation and levels of statistical significance in cases of analyses involving relationship between selected anthropometric and biochemical parameters, divided into two groups with anorexia nervosa (AN-I and AN-II)

\begin{tabular}{|c|c|c|c|c|c|}
\hline \multicolumn{2}{|c|}{ Parameter [unit] } & \multirow{2}{*}{$\begin{array}{c}\begin{array}{c}\text { Vaspin } \\
{[\mathrm{ng} / \mathrm{ml}]}\end{array} \\
\mathrm{NS}\end{array}$} & \multirow{2}{*}{$\begin{array}{c}\begin{array}{c}\text { Neuropeptide B } \\
{[\mathrm{ng} / \mathrm{ml}]}\end{array} \\
\mathrm{NS}\end{array}$} & \multirow{2}{*}{$\begin{array}{c}\begin{array}{c}\text { Neuropeptide W } \\
{[\mathrm{ng} / \mathrm{ml}]}\end{array} \\
\mathrm{NS}\end{array}$} & \multirow{2}{*}{$\begin{array}{c}\Delta \text { TAS } \\
r=0.48 \\
p<0.008\end{array}$} \\
\hline $\begin{array}{l}\text { AN-I } \\
(n=30)\end{array}$ & Body weight [kg] & & & & \\
\hline & $\begin{array}{l}\text { Vaspin } \\
{[\mathrm{ng} / \mathrm{ml}]}\end{array}$ & - & $\begin{array}{l}r=0.46 \\
p<0.02\end{array}$ & NS & NS \\
\hline & $\begin{array}{c}\text { Neuropeptide B } \\
{[\mathrm{ng} / \mathrm{ml}]}\end{array}$ & $\begin{array}{l}r=0.46 \\
p<0.02\end{array}$ & - & $\begin{array}{c}r=0.95 \\
p<0.000001\end{array}$ & NS \\
\hline \multirow[t]{3}{*}{$\begin{array}{l}\text { AN-II } \\
(n=30)\end{array}$} & Body weight [kg] & $\begin{array}{l}r=0.36 \\
p<0.05\end{array}$ & NS & NS & $\begin{array}{l}r=0.39 \\
p<0.04\end{array}$ \\
\hline & $\begin{array}{l}\text { Vaspin } \\
{[\mathrm{ng} / \mathrm{ml}]}\end{array}$ & - & $\begin{array}{c}r=0.45 \\
p<0.013\end{array}$ & NS & NS \\
\hline & $\begin{array}{c}\text { Neuropeptide B } \\
{[\mathrm{ng} / \mathrm{ml}]}\end{array}$ & $\begin{array}{l}r=0.45 \\
p<0.013\end{array}$ & - & $\begin{array}{c}r=0.97 \\
p<0.000001\end{array}$ & NS \\
\hline
\end{tabular}

Parameters are shown as $r$-coefficient of Pearson or Spearman (for respectively parametric or non-parametric date distributions) and $p$-level of statistical significance, $n$ - number of persons, TAS - total antioxidant status, $\triangle T A S$ - the change in TAS level in patients with anorexia nervosa (comparing status before and after hospital treatment), NS - statistically insignificant difference.

the same kind [NPB $(+/+)]$, but such changes were absent in the female population [25].

Furthermore, adult male mice, but not female mice, with a knockout gene for a specific receptor, namely GPR7 (the ligand for which is NPB), were characterized by increased leptin, insulin, and glucose levels related to hyperphagia and decreased physical activity [26]. The reasons for the differences in how NPB works between animals of different sex have not been explained. Among the human population, it is also not known why there is a higher prevalence of anorexia nervosa in the female than the male population. Disturbances in the levels of leptin in the blood of persons with anorexia nervosa indicate that fatty tissue plays a vital role in this disease. In the human body, the content of this tissue (which is the main deposit of leptin) is higher among females than males (even before puberty) [27].

As has been mentioned, the influence of neuropeptide $W$ on the human body is not fully understood. In vitro studies showed that NPW (in concentrations of $10-100 \mathrm{nmol} / \mathrm{l}$ ) increased insulin production from isolated pancreatic $\beta$-cells by way of an influx of $\mathrm{Ca}^{2+}$ ions via voltage-dependent L-type $\mathrm{Ca}^{2+}$ channels. This phenomenon took place with relatively high glucose levels $(8.3 \mathrm{mmol} / \mathrm{l})$, but such changes were not observed with $2.8 \mathrm{mmol} / \mathrm{l}$ [28].

Average TAS values in patients with anorexia nervosa only slightly changed after hospitalization. At the same time, changes of this parameter correlated positively with body weight measurements both in the $\mathrm{AN}-\mathrm{I}$ and $\mathrm{AN}-\mathrm{II}$ group. In a meta-analysis encompassing 7 clinical studies concerning patients treated for anorexia nervo$\mathrm{sa}$, it was noted that the level of albumin (anti- oxidant protein) and catalase activity increased, whereas another antioxidative enzyme, i.e., superoxide dismutase, and the concentration of apolipoprotein, which is a pro-oxidative indicator, decreased. Moreover, one of the studies found higher TAS levels after an average BMI increase of $2 \mathrm{~kg} / \mathrm{m}^{2}$ after hospitalization [19], which was not confirmed by the present research. It indicates that the mechanism of disturbances in pro- and antioxidative systems in anorexia nervosa is a complex one and perhaps other systems (i.e., neurobiological and endocrine) are more important than total antioxidant status.

To confirm the study results, it may be worth considering an extension of the research to include more female patients with anorexia nervosa, which is especially difficult in the case of this disease. This study is based on several weeks of observation of 30 patients with severe anorexia nervosa. When such a study is planned it needs to take into account the minimum sample size to enter the data into the statistical software, and this was done based on pilot study results. The assumed test power is 0.9 and type I error is 0.05 (parameters often used in medical studies).

In conclusion, the variety of determinants in restrictive eating habits in patients with anorexia nervosa indicates that the nature of the disorder is a complex one, and aside from a person's mental condition, also neurobiological and endocrine conditions need to be considered as causes of the disease and persistence of symptoms. Research results suggest that VASP plays a role in the normalization of anthropometric values during AN treatment. High values of NPB, despite treatment and normalization of VASP concentration, may 
indicate chronic neuroendocrine disorders in anorexia nervosa.

\section{Conflict of interest}

The authors declare no conflict of interest.

\section{References}

1. American Psychiatric Association. Diagnostic and Statistical Manual of Mental Disorders. Fifth Edition. Arlington, VA, American Psychiatric Association, 2013. Availabe: http://dsm.psychiatryonline.org/doi/full/10.1176/ appi.books.9780890425596.x00DiagnosticClassification [Cited 5 September 2017].

2. Fichter MM, Quadflieg N, Crosby RD, Koch S. Long-term outcome of anorexia nervosa: results from a large clinical longitudinal study. Int J Eat Disord 2017; 50: 1018-30.

3. Gorwood P, Blanchet-Collet C, Chartrel N, et al. New insights in anorexia nervosa. Front Neurosci 2016; 10: 256.

4. Grzelak T, Dutkiewicz A, Paszynska E, Dmitrzak-Weglarz M, Slopien A, Tyszkiewicz-Nwafor M. Neurobiochemical and psychological factors influencing the eating behaviors and attitudes in anorexia nervosa. J Physiol Biochem 2017; 73: 297-305.

5. Tortorella A, Brambilla F, Fabrazzo M, et al. Central and peripheral peptides regulating eating behaviour and energy homeostasis in anorexia nervosa and bulimia nervosa: a literature review. Eur Eat Disord Rev 2014; 22: 307-20.

6. Baranowska-Bik A, Baranowska B, Martynska L, et al. Adipokine profile in patients with anorexia nervosa. Endokrynol Pol 2017; 68: 422-9.

7. Kawai K, Nakashima M, Kojima M, et al. Ghrelin activation and neuropeptide $Y$ elevation in response to medium chain triglyceride administration in anorexia nervosa patients. Clin Nutr ESPEN 2017; 2: 100-4.

8. Yi J, Delp MS, Gilbert ER, Siegel PB, Cline MA. Anorexia is associated with stress-dependent orexigenic responses to exogenous neuropeptide Y. J Neuroendocrinol 2016; 28, doi: 10.1111/jne.12378.

9. Heiker JT, Klöting N, Kovacs P, et al. Vaspin inhibits kallikrein 7 by serpin mechanism. Cell Mol Life Sci 2013; 70: 2569-83.

10. Blüher M. Vaspin in obesity and diabetes: pathophysiological and clinical significance. Endocrine 2012; 41 : 176-82.

11. von Loeffelholz C, Möhlig M, Arafat AM, et al. Circulating vaspin is unrelated to insulin sensitivity in a cohort of nondiabetic humans. Eur J Endocrinol 2010; 162: 507-13.

12. Takenoya F, Wang L, Kageyama $\mathrm{H}$, et al. Neuropeptide $\mathrm{W}$-induced hypophagia is mediated through corticotropin-releasing hormone-containing neurons. J Mol Neurosci 2015; 56: 789-98.

13. Rucinski M, Nowak KW, Chmielewska J, Ziolkowska A, Malendowicz LK. Neuropeptide W exerts a potent suppressive effect on blood leptin and insulin concentrations in the rat. Int J Mol Med 2007; 19: 401-5.

14. Takenoya F, Kageyama H, Shiba K, Date Y, Nakazato M, Shioda S. Neuropeptide W: a key player in the homeostatic regulation of feeding and energy metabolism? Ann N Y Acad Sci 2010; 1200: 162-9.
15. Li H, Feinle-Bisset C, Frisby C, Kentish S, Wittert GA, Page AJ. Gastric neuropeptide W is regulated by mealrelated nutrients. Peptides 2014; 28: 6-14.

16. The ICD-10 classification of mental and behavioral disorders diagnostic criteria for research. 2003. WHO, Geneva. Available from: http://www.who.int/classifications/icd/en/ [Cited 5 September 2017].

17. Palczewska I, Niedzwiecka Z. Somatic development indices in children and youth of Warsaw. Med Wieku Rozwojowego 2001; 2: 108-18.

18. Khan N, Akhtar MS, Khan BA, et al. Antiobesity, hypolipidemic, antioxidant and hepatoprotective effects of Achyranthes aspera seed saponins in high cholesterol fed albino rats. Arch Med Sci 2015; 11: 1261-71.

19. Solmi M, Veronese N, Manzato E, et al. Oxidative stress and antioxidant levels in patients with anorexia nervosa: a systematic review and exploratory meta-analysis. Int J Eat Disord 2015; 48: 826-41.

20. Oswiecimska J, Suwala A, Swietochowska E, et al. Serum vaspin concentrations in girls with anorexia nervosa. J Pediatr Endocrinol Metab 2016; 29: 681-6.

21. Ostrowska Z, Ziora K, Oswiecimska J, et al. Vaspin and selected indices of bone status in girls with anorexia nervosa. Endokrynol Pol 2016; 67: 599-606.

22. Paszynska E, Slopien A, Tyszkiewicz-Nwafor M, Dutkiewicz A, Dmitrzak-Weglarz M, Grzelak T. How saliva is useful for identification of antioxidants and vaspin in anorexia nervosa. Eur Neuropsychopharmacol 2017; 27 (Suppl 4): S1104-5.

23. Vehapoglu A, Ustabas F, Ozgen TI, Terzioglu S, Cermik BB, Ozen OF. Role of circulating adipocytokines vaspin, apelin, and visfatin in the loss of appetite in underweight children: a pilot trial. J Pediatr Endocrinol Metab 2015; 28: 1065-71.

24. Tanaka H, Yoshida T, Miyamoto N, et al. Characterization of a family of endogenous neuropeptide ligands for the G protein-coupled receptors GPR7 and GPR8. Proc Natl Acad Sci USA 2003; 100: 6251-6.

25. Kelly MA, Beuckmann CT, Williams SC, et al. Neuropeptide B-deficient mice demonstrate hyperalgesia in response to inflammatory pain. Proc Natl Acad Sci USA 2005; 102: 9942-7.

26. Ishii M, Fei H, Friedman JM. Targeted disruption of GPR7, the endogenous receptor for neuropeptides B and W, leads to metabolic defects and adult-onset obesity. Proc Natl Acad Sci USA 2003; 100: 10540-5.

27. Tinggaard J, Hagen CP, Christensen AN, et al. Anthropometry, DXA and leptin reflect subcutaneous but not visceral abdominal adipose tissue by MRI in 197 healthy adolescents. Pediatr Res 2017; 82: 620-8.

28. Dezakia K, Kageyamab H, Sekib M, Shiodab S, Yadaa T. Neuropeptide $W$ in the rat pancreas: potentiation of glucose-induced insulin release and $\mathrm{Ca} 2+$ influx through L-type Ca2+ channels in beta-cells and localization in islets. Regulatory Peptides 2008; 145: 153-8. 